\title{
Mejora de habilidades en la seguridad del paciente implementando una estrategia de entrenamiento en el personal asistencial
}

\author{
D. Arias ${ }^{1}$, J.E Camacho ${ }^{2, \psi}$, J. Osorno ${ }^{1}$ \\ ${ }^{I}$ Universidad EIA- Universidad CES. Ingeniería Biomédica, Laboratorio Ingeniería Clínica, \\ Medellin, Colombia \\ ${ }^{2}$ Universidad EIA-Universidad CES. Docente-Investigador Ingeniería Clínica, Medellín, Colombia
}

\begin{abstract}
Resumen - En la actualidad, la sofisticación de la tecnología en el sector sanitario ofrece ventajas para el diagnóstico y tratamiento de los pacientes; sin embargo, los riesgos y eventos adversos que acompañan a esta nueva generación de tecnología son una tendencia creciente a nivel global. Hay muchos factores que contribuyen a este escenario, especialmente el error humano que aumenta en las instituciones de salud cuando el proceso de incorporación tecnológica no incluye la adecuada formación del personal. El objetivo de este proyecto es contribuir a la seguridad de los pacientes y ofrecer servicio de calidad en la atención sanitaria. Para ello debe implementarse una estrategia de capacitación del personal en el uso adecuado de la tecnología para ofrecer un servicio responsable, comprometido y profesional.
\end{abstract}

Palabras clave - Capacitación; dispositivo médico; seguridad del paciente; uso apropiado.

\section{Skill Improvement in Patient Safety through Training Strategy IMPLEMENTATION IN HEALTH-CARE WORKERS}

\footnotetext{
Abstract-Currently, the sophistication of technology in the health-care industry offers advantages for the diagnosis and treatment of patients; however, the risks and adverse events that go with this new generation of technology are a growing tendency worldwide. There are many factors that contribute to this scenario, but especially the increase in human error in health-care institutions when the process of technological incorporation does not include appropriate personnel training. The objective of this project is to contribute to patient safety and offer quality healthcare. In order to offer a responsible, committed, and professional service, a strategy of training personnel in the appropriate use of technology must be implemented..
}

Keywords — Training; Medical device; Patient safety; Appropriate use. 


\title{
MELHORA DE HABILIDAdES NA SEGURANÇA DO PACIENTE IMPLEMENTANDO UMA ESTRATÉGIA DE TREINAMENTO NO PESSOAL ASSISTENCIAL
}

\begin{abstract}
Resumo-Na atualidade a sofisticação da tecnologia no sector sanitário oferece vantagens para o diagnóstico e tratamento dos pacientes, no entanto, os riscos e eventos adversos que acompanham esta nova geração de tecnologias são uma tendência crescente a nível global. Existem muitos fatores que contribuem a este palco: especialmente o erro humano que aumenta nas instituições de saúde quando o processo de incorporação tecnológica não inclui a adequada formação do pessoal. O objetivo deste projeto é contribuir à segurança dos pacientes e oferecer atenção de qualidade na atenção sanitária, a implementação de uma estratégia de capacitação do pessoal no uso adequado da tecnologia para oferecer um serviço responsável, comprometido e profissional.
\end{abstract}

Palavras-chave - Capacitação; Dispositivo médico; Segurança do paciente; Uso apropriado.

\section{INTRODUCCIÓN}

$\mathrm{L}$ a Organización Mundial de la Salud (OMS) reconoce la importancia de los dispositivos médicos para la atención sanitaria y la mejora de la salud de las personas y las poblaciones [1]. Estos están designados a proceder directamente en el ser humano desde la intervención o la prevención de problemas de salud. Sin embargo, su implementación tiene incluidos componentes que atentan contra la seguridad de un paciente [2]. En Estados Unidos, el Instituto ECRI considera que los peligros en la tecnología médica pueden ocurrir, entre otros, por el manejo inadecuado por parte del personal, por la introducción de malos parámetros, malas configuraciones, inadecuados reprocesamientos, deficiencias en el mantenimiento y la mala gestión [3].

Así mismo la agencia FDA ha recomendado a los fabricantes mejorar las interfaces de usuario, pretendiendo así minimizar los errores durante el uso de este tipo de tecnología [4]. Por su parte la MHRA (Agencia Reguladora de Medicinas y Cuidado de la Salud) del Reino Unido, durante el periodo de 2011 a 2012 reportó 11.970 incidentes adversos relacionados con dispositivos médicos [5]. Frente a este nuevo reto del sector salud, diferentes expertos han discutido el impacto de los dispositivos médicos y efecto del factor humano en la seguridad del paciente y los riesgos que esto genera. Al respecto han propuesto diferentes estrategias para mejorar el uso de las tecnologías, entre ellas fortalecer el entrenamiento a los usuarios finales [6]. En las Instituciones de Salud (IS) de Colombia, la seguridad del paciente es una prioridad universal y al respecto se construyen iniciativas y estrategias que la fortalezcan. Una de ellas es la política de seguridad del paciente, la cual es impulsada por el Sistema Obligatorio de Garantía de Calidad en Salud (SOGCS), y pretende proteger al paciente, impactar en la mejora de la calidad y hacer más seguros los procesos de atención [7]. Al respecto, estrategias de entrenamiento han demostrado ser eficientes para minimizar los riesgos, los eventos adversos y aumentar la eficiencia de los dispositivos médicos.
Debido a la importancia de brindar una atención más segura y contribuir con la política de seguridad al paciente, se hace necesario proponer herramientas que propicien el uso eficiente, seguro y racional de las tecnologías en salud. Este artículo presenta el diseño de una estrategia de entrenamiento para el personal asistencial basado en la identificación de las mejores prácticas institucionales a través del benchmarking comparativo; posteriormente se selecciona una institución de salud para implementar la estrategia por medio de una prueba piloto, evidenciando así impactos positivos en la disminución de costos por correctivos y reportes de eventos adversos por mal uso de los equipos biomédicos.

\section{MetodologíA}

El estudio se realizó en tres etapas. La primera consistió en identificar a través de un benchmarking comparativo, aquellas instituciones de salud que evidenciaron las mejores prácticas y experiencias en entrenamiento al personal asistencial en el uso de equipos médicos a nivel regional. Para esto se definieron como criterios de selección los siguientes: a) instituciones con más de 50 camas, b) experiencia en gestión de la tecnología, c) acreditados en salud, d) mejores calificadas en Latinoamérica e) con tecnología de punta y/o f) con disposición en participar en el estudio. El estudio tuvo la participación de ocho (8) instituciones de salud en total, localizadas en dos (2) departamentos (Antioquia y Valle del Cauca).

Para la segunda etapa se realizó un análisis comparativo de las fortalezas de cada institución de salud participante, lo que sentó las bases para el diseño de una estrategia de entrenamiento que vincula lineamientos, herramientas y planes enfocados al personal asistencial. Además se recopiló la secuencia de pasos a seguir en el entrenamiento y las acciones que el personal debe conocer para el uso adecuado de la tecnología biomédica.

Por último se realizó una prueba piloto en una institución de salud del municipio de Itagüí, Antioquia, 
Colombia. En esta etapa se diseñaron los instrumentos de entrenamiento y evaluación para cuatro equipos biomédicos (bomba de infusión, desfibrilador, electrocardiógrafo $\mathrm{y}$ autoclave), los cuales fueron seleccionados con base en la criticidad del equipo para la institución, reportes de falla y necesidad de conocimiento en el manejo. Los entrenamientos se programaron para tener una duración de 40 minutos cada uno y tenían como objetivo fortalecer el desarrollo de las competencias del personal asistencial en el manejo adecuado de la tecnología biomédica. Como control del proceso, se diseñó una etapa de evaluación en la cual se verificó la comprensión de la información expuesta en el entrenamiento de cada equipo biomédico y una de seguimiento o rastreo para verificar las consecuencias que ha traído el entrenamiento en el personal, es decir, si se tienen resultados positivos o negativos después de este, si han realizado cambios que mejoren el uso del dispositivo, o si simplemente no hubo ningún cambio.

\section{Resultados}

\section{A. Benchmarking comparativo}

Se realizó un benchmarking comparativo en ocho IS, esto permitió identificar las fortalezas de los procesos de entrenamiento en uso de equipos médicos y su nivel de importancia. Este ejercicio permitió identificar que para el 75 $\%$ de las IS es altamente prioritario implementar estrategias de entrenamiento en el uso adecuado de la tecnología biomédica. El resultado se ilustra en la Fig. 1.

Las IS participantes también expresaron que una estrategia de entrenamiento podría impactar positivamente en los costos y la disminución de eventos adversos.

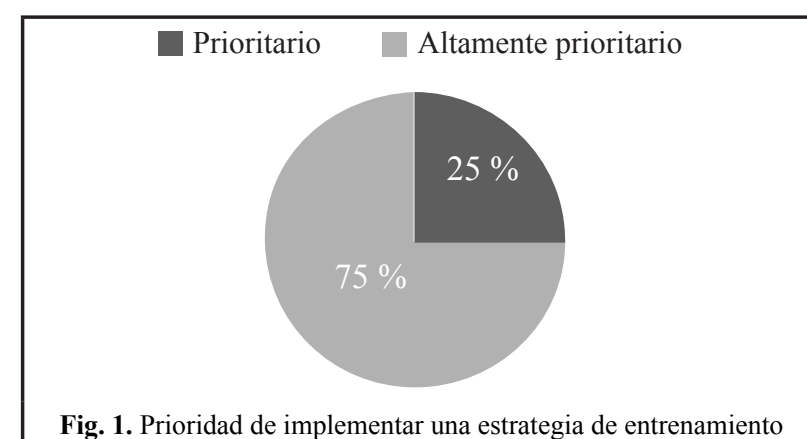

Fig. 1. Prioridad de implementar una estrategia de entrenamiento

Un análisis comparativo de los procesos identificados permitió evaluar cuantitativamente variables (la periodicidad, los cuidados y la información aplicada del fabricante, ver Tabla 1) y seleccionar de cada una de estas lo que se consideró más apropiado para construir una estrategia de entrenamiento de calidad.
Se determinó que la institución que posee el mejor proceso de capacitación, según parámetros establecidos en la matriz, es la Clínica del Prado de la ciudad de Medellín, esto debido a que su proceso se encuentra ampliamente implementado en todos los servicios, presenta resultados positivos frente a las metas propuestas y posee indicadores que lo respaldan. En las capacitaciones siguen las recomendaciones del fabricante para el buen uso y sistema de limpieza, además realizan estas con periodicidad (una vez al mes), por lo que se demuestra que en la institución las capacitaciones son un proceso constante.

\section{B. Estrategia de entrenamiento}

El diseño de una formación eficaz comienza con objetivos claros y específicos que son relevantes para el trabajo de los usuarios y el entorno de trabajo [8]. La estrategia vincula temas relevantes como gestión de alarmas en los equipos y la usabilidad por parte de las enfermeras [9]. El segundo requisito para un efectivo entrenamiento es que los usuarios deben tener la oportunidad adecuada para convertir su conocimiento intelectual en conocimiento funcional [6]. El tercer requisito es que deben definirse métricas y herramientas que permitan analizar la trazabilidad de la información aprendida. Es así como el personal asistencial, después de haber sido entrenado, debe demostrar el conocimiento y la habilidad para utilizar adecuadamente un dispositivo médico, y así quedar autorizados por la IS para usarlo con pacientes [8].

Con base en esto, se diseña una estrategia de entrenamiento simple y efectivo que permite desarrollar en el personal competencias para que su desempeño dentro de la organización sea más eficiente y seguro. En la Fig. 2 se ilustra la estrategia propuesta, la cual consta de la identificación de la necesidad, la definición de los equipos objeto de entrenamiento, el entrenamiento mismo en los temas priorizados, una evaluación y un seguimiento al proceso.

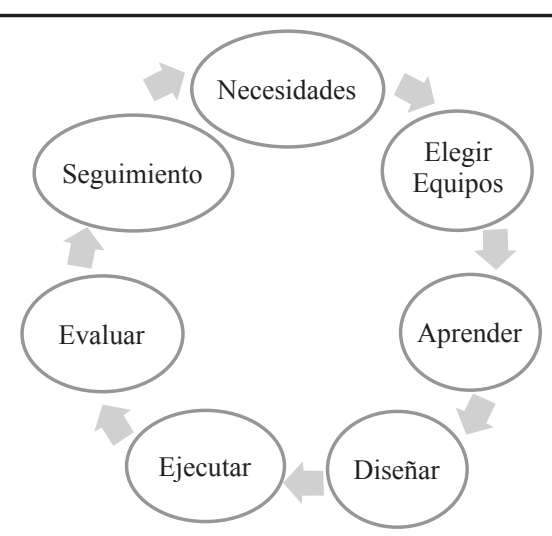

Fig. 2: Estrategia de entrenamiento 
Tabla 1. Análisis comparativo de los procesos identificados

\begin{tabular}{|c|c|c|c|c|c|c|c|}
\hline Institución & $\begin{array}{c}\text { Implementación } \\
\text { del proceso }\end{array}$ & $\begin{array}{c}\text { Evaluación del } \\
\text { proceso }\end{array}$ & Periodicidad & Duración & $\begin{array}{c}\text { Sugerencias del } \\
\text { fabricante }\end{array}$ & $\begin{array}{c}\text { Información } \\
\text { de cuidados y } \\
\text { limpieza }\end{array}$ & Valor \\
\hline $\begin{array}{l}\text { Hospital General de } \\
\text { Medellin }\end{array}$ & 3 & 3 & - & 3 & 4 & 3 & 3,2 \\
\hline $\begin{array}{c}\text { ESE Hospital del } \\
\text { Sur-Itagüi }\end{array}$ & 3 & 5 & 3 & 4 & 4 & 4 & 3,8 \\
\hline $\begin{array}{l}\text { Clínica del Prado } \\
\text { de Medellín }\end{array}$ & 5 & 5 & 5 & 4 & 5 & 5 & 4,8 \\
\hline $\begin{array}{c}\text { Clínica Palma Real } \\
\text { de Palmira }\end{array}$ & 5 & 2 & 5 & 4 & 5 & 5 & 4,3 \\
\hline $\begin{array}{l}\text { Clínica CES de } \\
\text { Medellín }\end{array}$ & 3 & 2 & 3 & 4 & 4 & 5 & 3,5 \\
\hline $\begin{array}{c}\text { Fundación Valle de } \\
\text { Lili-Cali }\end{array}$ & 4 & 3 & 5 & 4 & 5 & 5 & 4,3 \\
\hline $\begin{array}{c}\text { Centro clínico Sicor } \\
\text { Medellin }\end{array}$ & 3 & 2 & 3 & 4 & 4 & 4 & 3,3 \\
\hline
\end{tabular}

Tabla 2. Implementación de la estrategia

\begin{tabular}{|c|c|c|c|c|c|c|}
\hline Tema & Tecnología & Necesidades & Personal & Asistentes & Evaluación & Logros \\
\hline Autoclave & $\begin{array}{l}\text { Automat } 2400 \\
\text { Dental x ray }\end{array}$ & $\begin{array}{l}\text { Soportes frecuentes, errores } \\
\text { de uso, descontentos con la } \\
\text { tecnología }\end{array}$ & $\begin{array}{l}\text { Odontólogos } \\
\text { Auxiliares } \\
\text { odontología }\end{array}$ & 8 & $87,50 \%$ & $\begin{array}{l}\text { Conocen las instrucciones } \\
\text { correctas de uso, cómo } \\
\text { solucionar los posibles errores. }\end{array}$ \\
\hline Desfibrilador & $\begin{array}{l}\text { Beneheart D6 } \\
\text { Mindray }\end{array}$ & $\begin{array}{l}\text { Desconocimiento de todas } \\
\text { las partes físicas. Uso } \\
\text { incorrecto de gel }\end{array}$ & Médicos & 17 & $92,2 \%$ & $\begin{array}{c}\text { Reconocen el uso correcto, las } \\
\text { partes físicas del equipo y el } \\
\text { gel adecuado para usar. }\end{array}$ \\
\hline Electrocardiógrafo & CM300 Comen & $\begin{array}{c}\text { Soportes frecuentes, errores } \\
\text { de uso }\end{array}$ & $\begin{array}{l}\text { Enfermeras y } \\
\text { auxiliares de } \\
\text { enfermería }\end{array}$ & 9 & $96,42 \%$ & $\begin{array}{c}\text { Conocen las instrucciones } \\
\text { de funcionamiento, el gel } \\
\text { adecuado de uso. }\end{array}$ \\
\hline Bomba de infusión & $\begin{array}{l}\text { SK-600II } \\
\text { Mindray }\end{array}$ & $\begin{array}{c}\text { Uso permanente, } \\
\text { descontentos por parte del } \\
\text { personal }\end{array}$ & $\begin{array}{l}\text { Enfermeras y } \\
\text { auxiliares de } \\
\text { enfermería }\end{array}$ & 17 & $90,20 \%$ & $\begin{array}{l}\text { Conocen el uso correcto, } \\
\text { los posibles errores y cómo } \\
\text { solucionarlos. }\end{array}$ \\
\hline
\end{tabular}

\section{Implementación de la estrategia}

En esta fase del estudio se realizó una prueba piloto en una institución de salud del Municipio de Itagüí, Antioquia. Para esto se diseñaron instrumentos de entrenamiento y evaluación siguiendo los lineamientos definidos en la estrategia; se eligieron cuatro equipos biomédicos: bomba de infusión, electrocardiógrafo, desfibrilador y autoclave.

La estrategia de entrenamiento se implementó con el personal asistencial de los servicios de odontología y urgencias. Se ilustra en la Tabla 2 la información de los equipos biomédicos seleccionados, las necesidades particulares que se diagnosticaron en cada tecnología, público objetivo, número de asistentes, promedio de preguntas aprobadas en la evaluación realizada y los logros obtenidos durante la formación.

La prueba piloto se realizó en un periodo de dos meses y permitió aumentar el nivel de conocimiento del buen uso de los equipos médicos en un $90 \%$. El personal que se sometió al entrenamiento se involucró con la estrategia, generando impactos positivos en los indicadores internos de la institución tales como una reducción de un 30\% de reportes de mantenimientos correctivos, aumentó la usabilidad de los equipos en un $50 \%$ y no se generaron reportes de mal uso de estos durante el tiempo de la medición.

Además, la estrategia sirvió de apoyo en el cumplimiento del estándar de gestión de tecnología para el proceso de acreditación en salud, beneficiando a la institución en la obtención de la renovación de la acreditación.

Por último se desarrolló un documento con la información más relevante que debe conocer el personal para que haga correcto uso del equipo, enfocado en el manual de usuario de cada uno de los dispositivos.

También se crearon videos y presentaciones con la información de las partes físicas, funciones de los botones e instrucciones de operación para el uso seguro. 
Adicionalmente se dispuso esta información en forma digital en la web a través de la construcción de un blog usando la aplicación de Google Blog, la dirección electrónica es http://trainingbiomedicaldevice.blogspot.com.co/. De esta forma los usuarios pueden acceder a consultar el material de entrenamiento durante las 24 horas, siendo esta una herramienta para prevenir problemas por desconocimiento de la tecnología.

\section{Conclusión}

Se desarrolla una estrategia de entrenamiento en el uso adecuado de la tecnología biomédica para el personal implicado en la atención de la salud, contribuyendo así en la generación de servicios asistenciales más seguros, con responsabilidad, compromiso y profesionalidad. Así mismo se contribuye con una herramienta que apoya el cumplimiento de la política de seguridad del paciente. Se concluye por lo tanto que estrategias de entrenamiento ajustadas a las necesidades de las instituciones de salud contribuyen en el uso adecuado de los equipos biomédicos, por ende se disminuyen los eventos adversos, así como la incidencia de quejas y requerimiento de soportes, los costos de mantenimientos correctivos son menores y además se aumenta la vida útil de estos; todo lo anterior redunda en la calidad de la atención prestada a los pacientes.

Igualmente, crear en las instituciones de salud la cultura de capacitación, ya que existe la necesidad permanente de adquirir competencias para mejorar el desempeño del personal, además de que todos los individuos requieren una actualización de conocimientos para avanzar tanto profesionalmente como personalmente.

Para la IS donde se realizó la prueba piloto, se entrenaron en total 51 personas y se definieron las oportunidades de mejora en el proceso de capacitación establecido con respecto a las otras siete entidades comparadas. Se recomienda que el entrenamiento al personal no sea algo ocasional sino un proceso continuo, impulsando el fortalecimiento de los procesos de capacitación en las instituciones para mejorar el nivel de rendimiento presente y futuro del personal que labora en estas.

\section{Conflicto De InTERESES}

Los autores declaran que no hay conflicto de intereses en la producción de este artículo.

\section{Agradecimientos}

Los autores agradecen a las instituciones de salud: Hospital General de Medellín, a la Clínica del Prado, clínica Palma Real, Clínica CES, Fundación Valle de Lili, San Vicente Fundación, al Centro Clínico y de Investigación
Sicor. Igualmente, a la Ingeniera Natalia Tabares Franco y al ingeniero Santiago Marín Echeverri por participar en benchmarking y a la ESE Hospital del Sur de Itagüí por facilitar el desarrollo de la prueba piloto.

\section{REFERENCIAS}

[1]. OMS. Dispositivos médicos: Gestión de la discordancia, 2012.

[2]. INVIMA, «INVIMA,» (2012). Consultado en 2015 en: https://www.invima.gov.co/component/content/article. html?id=768:seguridadde-los-dispositivos-medicos.

[3]. ECRI Institute. El Hospital (junio 2015). Consultado el 27 de septiembre de 2015 en: http://www.elhospital.com/temas/ Los-10-primeros-riesgos-de-latecnologia-medica-para-el-2015Parte-1+105791

[4]. Food and Drug Administration. Applying Human Factors and Usability Engineering to Medical Devices. 2016.

[5]. MHRA. «MHRA,» (2011). Consultado el 20 de septiembre de 2015 en: http://www.fdanews.com/ext/resources/files/archives/c/ con129234.pdf.

[6]. Cassano A., Trbovich P., Griffin M., Ling L., Easty T. Human factors for health technology safety: Evaluating and improving the use of health, 2015.

[7]. Ministerio de Salud. Lineamientos para la implementación de la seguridad del paciente en la República de Colombia. Bogotá, 2008.

[8]. Hyman W. A practicum for biomedical engineering \& technology management Issues, pp. 141-154, 2008.

[9]. Honan L., Funk M., Maynard M., Fahs D., Clark T., David Y. Nurses' Perspectives on Clinical Alarms. American Journal of Critical Care, 24(5), pp. 387-395, 2015. 